In Crescendo. Institucional. 2016; 7(1): 134-144

Fecha de recepción: 6 de mayo de 2016

Fecha de aceptación: 17 de mayo de 2016

\title{
CONSULTORIOS DE ENFERMERÍA. UNA OPORTUNIDAD PARA GENERAR DESARROLLO HUMANO Y LOCAL
}

\section{Nursing CLINICS. AN OPPORTUNITY TO GENERATE HUMAN DEVELOPMENT AND LOCAL}

\section{Fredy Hernán Polo Campos*}

$E^{1}$ presente ensayo tiene por objetivo posicionar el debate sobre la autonomía en el ejercicio profesional de enfermería dentro de un contexto de lucha que viene afrontando la clase trabajadora de enfermeras y enfermeros por conseguir la aprobación de la Ley 35-2011, que crea y reglamenta la creación de los consultorios de enfermería como espacio físico donde los profesionales de la salud proporcionan un servicio independiente y autónomo. Para alimentar este debate, empezaremos haciendo una reflexión crítica sobre la necesidad de una política social que genere desarrollo humano y social, siendo esta, una aspiración legítima de las personas y de sus colectivos sociales de tener acceso al bienestar y a la autonomía. En correspondencia, el Estado y la sociedad civil deben concordar en la necesidad de implementar un modelo de política pública que estimule un proceso de expansión de las libertades en individuos y colectivos sociales como base para el desarrollo (Sem, 2000).

Primeramente, sostenemos que la política sanitaria actual se encuentra en crisis, pero una crisis de desarrollo, esta se evidencia en el incremento de las demandas de prestación de servicios en número, diversidad y calidad, sin embargo, la oferta de servicios público-privados no ha crecido en la misma proporción, más aún cuando esta no reúne criterios mínimos de calidad e intensidad que se requiere. En justa medida, la política sanitaria requiere de un nuevo modelo de prestación que atienda la demanda sentida y que a la vez genere desarrollo.

En correspondencia a esta primera atingencia, proponemos la necesidad de implementar un nuevo modelo sanitario, un modelo que privilegie el cuidado de la vida-salud y de la libertad-autonomía, fines supremos de toda política pública responsable (Polo

\footnotetext{
* Enfermero epidemiólogo-salubrista, exsubgerente de cuidado integral de salud del Gobierno regional de la Libertad, Perú. Doctor por la Universidad Federal de Río de Janeiro, profesor asociado de la Universidad Nacional de Trujillo, Perú. Docente invitado de posgrado y postítulo en la Universidad Santo Toribio de Mogrovejo, Chiclayo, Perú. Actualmente, posdoctorando por la Universidad Federal de Río de Janeiro, Brasil.
} 
Campos, 2009). La característica del modelo de cuidado, a diferencia del modelo de atención de salud que genera dependencia, asistencia y tiene como foco al "paciente", este genera desarrollo de competencias de las personas y sus colectivos (familia, instituciones educativas, centros laborales, barrios, comunidades, etc.) a fin de tornarlo autosuficiente, constructores de su bienestar y del ejercicio de ciudadanía.

En el país, existen experiencias en la implementación de un modelo de cuidado de la salud y desarrollo de autonomía en el sistema de servicios públicos. La experiencia en la región La Libertad nos dice que consolidar una nueva práctica sanitaria requiere, no solo un cambio paradigmático en la gestión y prestación de los servicios, sino que, además se necesita una estructura que permita hacer tangible la oferta de servicios de cuidado humano (Polo Campos, 2010).

En el sistema privado no se reportan experiencias de estructuras que permitan la oferta de servicios de cuidado, en contraparte son los profesionales de enfermería los que reportan más investigaciones respecto a modelos de cuidado de la salud, sin duda esta nos ha sido estimulada por las restricciones y limitaciones legales que se tiene para el ejercicio de una práctica profesional libre y autónoma. Sin embargo, existen iniciativas legales a la modificación de la ley 27669 (Ley del Trabajo del Enfermero) a fin de dotarle de autonomía para el ejercicio profesional en el ámbito público y privado.

Teniendo como necesidad la implementación de un modelo de política de cuidado de la salud como base para generar desarrollo humano y social, además del reconocimiento que los enfermeros (as) son los profesionales que más competencias intelectuales y técnicas ha desarrollado sobre el cuidado, en consecuencia, la creación y reglamentación de los Consultorios de enfermería (Ley 35-2011) se hace necesaria y constituye una importante estrategia socio-sanitaria que el Estado-Sociedad Civil debe promover.

Los consultorios de enfermería son necesarios para fortalecer la autonomía profesional, sin embargo, es necesario destacar su potencial contribución en mejorar la calidad de vida de la persona, de la familia y la comunidad. Sin duda para los enfermeros y enfermeras tiene una simbología importante en la medida que representa el espacio del ejercicio autónomo y consecuentemente fortalece la identidad profesional. Es bueno resaltar que se precisa no solo de estructura, sino de un cuerpo propio de conocimientos especializados, que sustente una oferta de servicios diferenciados en el sistema público y privado.

Modelar una política de cuidado de la salud como base para el desarrollo humano y local, sobre la base de la creación y reglamentación de los consultorios de enfermería, genera responsabilidades como gremio especializado en proporcionar cuidado, las discusiones teóricas-metodológicas realizadas en las escuelas de postgrado y segunda especialización sobre el sistema sanitario, nos permite visualizar algunas debilidades existentes y avizorar amenazas en el proceso de consolidación de una práctica autónoma para la enfermería.

No podemos negar que el proceso profesionalizante de la enfermería en el país condujo a tener una representación social de una profesión eminentemente práctica (Hatton, E. J., 1995), esta es un legado que tenemos que superar o suplantar. En efecto, históricamente, el conocimiento experto que sustentaba nuestra práctica fue producto de un prolongado proceso de socialización, se ha transmitido de generación en generación acumulándose 
progresivamente, adquiriendo un carácter sistémico que no favorece a la autonomía, reduciéndola a una práctica conservadora, que limita la creatividad y, sobre todo, lo más riesgoso se evidencia la ausencia de una concepción teórica sobre su trabajo (Pérez A. J., 1993). Esta representación social aun nos atinge, aunque confieso con alegría en menor escala gracias a la contribución de la academia y la investigación.

Un segundo aspecto, no menos preocupante, es el rol y desempeño del Estado, en especial del interés de sus gobernantes que colocan contenidos políticos encubiertos, establecen una legislación para conducir comportamientos y determinar estructuras legitimando su hegemonía. La crisis del modelo de atención flexneriano que aducíamos en su inicio está sustentado en un enfoque biologista, que privilegia las acciones recuperativas y rehabilitadoras.

No es gratis el interés de los colegios profesionales de las carreras relacionados con la salud; de estar en contra de una práctica sanitaria que promueva el desarrollo humano; y de estrategias como los consultorios de enfermería. Ellos defienden un paradigma positivista, un estatus político-social y un poder económico. Tras de ellos están las grandes industrias farmacéuticas, los grandes consorcios que operan servicios de ayuda al diagnóstico, cuyo interés legítimo o no, es el lucro en un mercado inequitativo como es el de salud. Es interés del Estado-ciudadano, fortalecer el cuidado preventivo y promocional que oferta la consulta de enfermería.

Este contenido político encubierto genera una obsesión por los métodos y recursos instrumentales; todos los procedimientos son controlados y estandarizados, esta situación erosiona la especificidad que asiste al cuidado profesional, más aún porque tratamos con personas. El exceso interés por reglamentar los procedimientos, limita el ejercicio sensible y creativo de la práctica de enfermería, tornando al profesional de la salud un burócrata eficaz sometido a un aparato administrativo. En consecuencia, la política actual genera una práctica impuesta con técnicas predecibles y discursos teóricos muchas veces construidos sin tener en cuenta las necesidades de nuestro trabajo.

Nuestro sistema sanitario está plagado de estos ejemplos, uno de ellos es el uso de indicadores negativos para determinar el estado de la salud como la tasa de mortalidad, letalidad, etc., serviría muy bien para determinar el estado mórbido o de muerte en una población, mas no de la salud. Indicadores de salud: niño sano, adolescente con proyecto de vida, adulto con enfermedad crónica controlado, comunidad pacificada, etc., podría representar un perfil de salud. Otro ejemplo, es la asignación inequitativa de presupuesto para actividades preventivas promocionales, nuestro país invierte no más del 10\% en esta actividad y se denomina ministerio de la salud, mejor sería denominado ministerio que atiende la enfermedad o en el mejor de los casos que recupera la salud.

Haciendo una lectura crítica de la realidad respecto a la creación y reglamentación de los Consultorios de Enfermería, hay un interés antiprofesional de otras carreras de las ciencias de la salud que quieren seguir manteniendo una hegemonía y control del mercado de la salud (Iglesias, 1995). En resumen, la autonomía está amenazada, siempre la estuvo, por este tipo de interés y nos corresponde combatirla con conocimiento, (por nues- 
tros usuarios y por nosotros mismos) estos modelos que buscan constreñir y no permiten cultivar la autónoma como base del ejercicio profesional autónomo (Polo Campos, 2009).

El contexto socio-político en que vivimos, también ofrece oportunidades y fortalezas que aprovechar. En efecto, se observa una gran expansión del área de actuación del enfermero. Este proceso que se caracteriza por una redefinición del límite de desempeño profesional, acompañado por una mayor autonomía en el ejercicio de sus actividades. En la XIII conferencia de educación en enfermería celebrada en Río de Janeiro en el 2015, discutimos el tema, resaltando la necesidad de establecer responsabilidades y límites de actuación en cada punto de entrega de servicios (consultorios de enfermería) el mismo que debe estar amparado en la ley y ser de conocimiento del usuario para su demanda.

Existen aún vacíos legales sobre los límites de actuación del enfermero(a) sobre todo en América-Latina. Experiencias en países como Reino Unido, Estados Unidos y Canadá, revelan que esas atribuciones ya están mejor establecidas y el enfermero es cualificado para tratar una gran variedad de enfermedades. Ejemplos: incluyen las mordidas de animales, conjuntivitis, eczema, cistitis, impétigo, úlceras en la cavidad bucal y otras. Pueden prestar además una serie de servicios especiales como curativos, vacunación infantil, consejos de salud a los padres de niños pequeños y orientación para planificación familiar. También proporcionan cuidados de rutina y solicitan exámenes complementarios, para una serie de problemas crónicos, tales como asma, diabetes, hipertensión. Y hacen evaluaciones anuales en pacientes víctimas de infarto o accidente vascular encefálico (Cárnio, 2013).

Otra fortaleza la constituyen los manifiestos y reconocimientos de los organismos internacionales especializados en salud. La OMS-OPS, reconocen que la "misión de los servicios de enfermería es prestar atención de salud a las personas, familias y comunidades en todas las etapas del ciclo de vida y en los diferentes aspectos de prevención de enfermedades, promoción de la salud, recuperación y rehabilitación con enfoque holístico e interdisciplinario con el fin de contribuir al mayor bienestar de la población" (OMS, 2010).

El marco legal, Ley 27669, art. 7, inciso d, donde se refiere que los enfermeros(as) tienen la función de ejercer consultoría, auditoría, asesoría y consejería, en mérito a ello, el ejercicio profesional de enfermería en los consultorios es viable jurídicamente y legítimo porque es aspiración de una categoría de trabajadores y de enormes beneficios para el desarrollo humano y social. El Proyecto de Ley 35-2011, modifica los artículos 3. ${ }^{\circ}$ y 9. ${ }^{\circ}$ de la Ley N. ${ }^{\circ}$ 27669, Ley de Trabajo del Enfermero(a), cuenta con dictamen favorable, y establece en uno de sus párrafos que "Enfermería, debe y tiene el derecho de ejercer la profesión a través de consultorios privados dentro de su competencia" entendiéndose este como el espacio físico dentro o fuera de un Establecimiento de Salud público o privado.

Una fortaleza lo constituye el hecho que Enfermería tiene una sólida formación universitaria, con escuelas y facultades acreditadas o en proceso de acreditación; sin duda alguna la formación universitaria proporciona competencia cognitivas, procedimentales y actitudinales para el ejercicio de la profesión de forma autónoma e independiente. 
La investigación está generando constantemente conocimiento experto (dígase de disciplinario) y pensamiento crítico la misma que es insumo para crear, controlar y recrear procesos de intervención individual y colectiva en favor del bienestar y el ejercicio de autonomía. Este hecho genera derecho de ser un profesional competitivo en los servicios públicos y privados (Iglesias, 2015).

El consultorio de enfermería es definida como un espacio físico donde el usuario demanda una consulta según su necesidad. Entiéndase como consulta de enfermería al proceso de interacción directa, donde el enfermero(a) oferta cuidados de promoción de la salud, prevención de riesgo, recuperación y rehabilitación de la enfermedad o discapacidad a sujetos-objetos y sus colectivos sociales, de forma integral, con calidad y universal.

Establecer límites y responsabilidades en el cuidado de la salud y desarrollo de autonomía, implica aprovechar la oportunidad de definir los servicios a ofertar y productos sociales a conseguir como efectos directos de nuestro ejercicio profesional que se caracteriza por ser intelectual y autónomo. En esta perspectiva, es necesario definir como nuestro sujeto-objeto de intervención: la persona y sus colectivos sociales como la familia, institución educativa, centro laboral, el barrio, la comunidad, etc.; asimismo, es necesario caracterizar el tipo de cuidados a ofertar según necesidad en cada nivel de atención.

A diferencia de otras disciplinas, nosotros atendemos a personas y colectivos sociales entre ellos las familias y comunidades conformadas por ciudadanos con deberes y derechos socialmente reconocidos y a la vez son objeto de nuestra intervención, es el pensamiento existencial de Heidegger diríamos: es con él y a través de él sujeto-objeto que el Enfermero consigue ser trascendente. Las discusiones teóricas nos llevan a establecer algunas características del cuidado auténtico (Heidegger, 2009) definido como el proceso que permite el desarrollo de competencias del ser para cuidar de sí; lo opuesto es hacer por el otro, lo que podría hacer por sí mismo. El cuidar auténticamente implica conectividad, receptividad y reciprocidad, características esenciales del proceso de cuidar.

Respecto al tipo de cuidados a ofertar, esta se determina según la oportunidad de intervención que el usuario (persona/colectivo) permite; y según sea el motivo de la consulta, puede ser cuidados rehabilitadores, recuperativos, preventivos y promocionales; incluso según los antropólogos cuidamos del duelo y del tránsito que la persona recorre hasta la muerte. A continuación haremos un esfuerzo por diferenciar y caracterizar los tipos de cuidado que enfermería precisa ofertar.

Los cuidados rehabilitadores están enfocados a la prestación de servicios a personacolectivos que presentan un problema causado directamente por: la insatisfacción de una necesidad; una enfermedad; un trastorno; o cualquier otra alteración de la salud y la autonomía que requiera asistencia rehabilitadora en forma de tratamiento individualizado y/o colectivo.

El manejo de las consecuencias de una carencia, de una enfermedad está dirigido a facilitar la adaptación de la persona a su nueva situación para desempeñarse de mejor manera en el diario vivir. Cuidados que faciliten la adaptación, el aprendizaje de técnicas compensatorias y el desarrollo de su autonomía son fundamentales para desarrollar competencias en el desempeño de sus roles personales-sociales mejorando su calidad de vida. 
El cuidado rehabilitador debe estar orientados a la atención de factores potenciales biopsicosociales que afecten directa o indirectamente. Los factores biológicos como: el diagnostico subyacente, pronostico estimado, síntomas mayores, otros problemas y/o necesidades de salud podrían estar potenciando o limitando la rehabilitación; factores psicológicos como: estado de ánimo, motivación, nivel de conciencia, etc., cuando son abordados adecuadamente conllevan a progresos significativos en la rehabilitación; y factores sociales como: soporte y cohesión familiar, estatus económico, cuidador familiar, redes sociales, oportunidades de re-admisión, influyen significativamente potenciando o limitando el éxito de los cuidados rehabilitadores (Frank et al., 1998).

Los usuarios que demandan este tipo de cuidados presentan secuelas, discapacidad temporal o permanente, en este sentido, se debe organizar la oferta de servicios e implementar estrategias de prevención terciaria, tendientes a limitar o prevenir la severidad del daño o evento de salud. La oportunidad y la intensidad de usos de estos servicios determinará su rehabilitación y discapacidad permanente del usuario; además de aliviar la carga social y financiera de familia; disminuirá el gasto en infraestructura, equipos y recurso humano por parte del Estado.

Los cuidados recuperativos tienen su foco de atención en la restauración de la salud. La recuperación se define como un proceso "muy personal" y único que supone el desarrollo de un nuevo significado y propósito en la vida más allá de los efectos negativos de la enfermedad. Se trata de un proceso continuo, que consiste en adquirir o recuperar muchos aspectos de la vida cotidiana de las personas, que pueden haberse perdido a causa de la enfermedad o evento adverso.

Las prácticas de cuidado orientadas a la recuperación tratan de mantener la calidad de vida, haciendo hincapié en la capacidad de una persona-colectivo a tener esperanza y desarrollar una vida con sentido para ella misma, según sus metas y ambiciones. El enfoque de recuperación parte de la base de que se obtendrán mejores resultados si las personas sienten que las decisiones sobre su tratamiento se realizan de acuerdo a sus ideas culturales y valores personales. El enfoque de recuperación se centra en el bienestar y la resistencia a la enfermedad, y alienta a las personas a participar activamente en su propio proceso de autocuidado, lo que permite que ellas mismas definan sus objetivos vitales y de tratamiento farmacológico, psicológico y social (Astudillo, s./f.)

Los cuidados recuperativos centran su foco de intervención en evitar y prevenir la aparición de las complicaciones producto del proceso mórbido de la enfermedad o evento de vida; para ello los cuidadores deben organizar el servicio e implementar estrategias de prevención secundaria. El diagnóstico oportuno y el tratamiento adecuado son las principales herramientas que permiten evitar las complicaciones con efectos innecesarios en la calidad de vida.

Existe mucha evidencia científica respecto a los cuidados recuperativos, generalmente se ofertan dentro de los servicios de salud en los diferentes niveles de atención, en consecuencia, el gestor del cuidado organizará la respuesta social según capacidad resolutiva. Los cuidados recuperativos son más frecuentes en el II y III nivel de atención, sin embargo, la oportunidad en el diagnóstico y el inicio temprano del tratamiento son vitales sobre 
todo en el control de enfermedades transmisibles y no transmisibles; en las enfermedades mentales y en los problemas sociales como violencia y pandillaje.

La prevención es una medida o disposición que se toma de manera anticipada para evitar que suceda una situación considerada negativa. La prevención, por la tanto, es la disposición que se hace de forma anticipada para minimizar un riesgo. El objetivo de prevenir es lograr que un perjuicio eventual no se concrete, por lo tanto, es mejor invertir en prevención que en un tratamiento paliativo. La OMS, define a la prevención primaria como "medidas orientadas a evitar la aparición de una enfermedad o problema de salud mediante el control de los factores causales y los factores predisponentes o condicionantes". Las estrategias para la prevención primaria pueden estar dirigidas a prohibir o disminuir la exposición del individuo al factor nocivo, hasta niveles no dañinos para la salud.

Denominaremos prevención primaria, como el proceso que conlleva a mitigar factores de riesgo que puedan causar lesiones, antes de que sean efectivos, antes de que se instale el proceso mórbido o evento; su principal objetivo es disminuir la incidencia de la enfermedad. En consecuencia, discrepamos de la definición de la OMS y postulamos que en el cuidado preventivo, lo que se previene es el riesgo a través de la intervención de sus causas (factores de riesgo), no se previene la enfermedad porque ella existe, se previene el riesgo de enfermar o morir, su ámbito de intervención es en la etapa prepatogénica.

Los cuidados preventivos son de vital importancia, en la medida que proporciona una oportunidad inmensurable al sistema sanitario para preservar la salud a través del control de los factores de riesgos. Los cuidados preventivos como estrategia contra-hegemónica, sin duda es la principal herramienta para desarrollar competencias en la sociedad civil, haciéndola auto-responsable de su vida-salud, tornándola promotora de su libertad- autonomía que son los principios básicos de toda política sanitaria. Es en este espacio donde se establecen las nuevas relaciones de poder en torno al cuidado libertador (Polo Campos, 2010).

Finalmente, definimos que los cuidados promocionales, como estrategia de desarrollo humano y local son de suma importancia, en la medida que permite incrementar el control sobre su salud-autonomía para mejorarla-desarrollarla, a diferencia de la prevención donde el foco es prevenir y minimizar riesgos. La definición dada en la histórica Carta de Ottawa (Ministerio de Salud de México, 1986) refiere que la promoción de la salud "consiste en proporcionar a la gente los medios necesarios para mejorar la salud y ejercer un mayor control sobre la misma". En correspondencia, la carta recomienda: construir políticas públicas saludables, crear ambientes que favorezcan la salud, desarrollar habilidades personales, reforzar la acción comunitaria, y reorientar los servicios de salud.

Operacionalizar el cuidado promocional conlleva la reorientación de los servicios públicos y privados tornándolos genuinamente establecimiento de salud y no como son ahora establecimientos de la enfermedad o para atender la enfermedad debido al énfasis del cuidado recuperativo rehabilitador. En este contexto, los cuidados promocionales tienen como foco, el desarrollo de competencias individuales y colectivas, el empoderamiento de la sociedad civil tornándolo autodeterminado. Este tipo de cuidados son ofertados especialmente a la población sana o aparentemente sana y centra su atención en desarro- 
llar competencias cognitivas, procedimentales y actitudinales en la persona-colectivo que garantice un desarrollo digno y pleno estado de bienestar.

Respecto al cuidado de los colectivos sociales, se destaca un interés creciente de demanda de cuidado al objeto-sujeto familia, por parte del sistema sanitario. En la actualidad, es una prioridad de atención en la política de salud, sin embargo, se evidencia una escasa respuesta por parte de los servicios de salud en la medida que no cuentan con recursos suficientes para operar. En esta medida los programas de segunda especialización en salud familiar y comunitaria desarrollados en el norte del país, están contribuyendo con discusiones críticas sobre la salud familiar, además de proporcionar proyectos de desarrollo para la creación de consultorios de enfermería, porque estamos convencidos que su funcionamiento constituyen una oportunidad de oferta de servicios de cuidado que la familia necesita.

En el modelo de cuidado de la salud, la familia, es definida como un "ser vivo" único, que tiene un inicio, se desarrolla, crece y tiene un fin que en muchos casos es el inicio de otra familia, el enfoque sistémico de etapas de vida familiar, permite describir las diferentes necesidades y problemas que afronta. La salud familiar, se constituye en un proceso de cuidado cuyo producto es más que la suma de la salud de sus miembros, está determinada por el desarrollo de competencias colectivas que garantice gozar de vida-salud y de libertad-autonomía.

En consecuencia, cuidar de la familia implica ofertar servicios no solo orientados a la atención de sus problemas que afecten la salud y autonomía familiar, sino por el contrario, y sobre todo, estimular las competencias del colectivo con visas a lograr disfrutar de las bondades del tan anhelado desarrollo. En cada etapa de vida la familia se presenta necesidades de cuidado: en la etapa de formación de pareja, de ser padres, en el crecimiento de los hijos, en la familia con hijos adolescentes, el dilema de dejar ir a los hijos que se emancipan, y la llegada de la etapa tardía donde la pareja se queda nuevamente sola marcan nuestra intervención. La oferta de estos cuidados muy bien puede ser proporcionado desde los consultorios de enfermería, con una visión interdisciplinar atendiendo a dos aspectos importantes: la primera por constituirse en la puerta de entrada del sistema sanitario; y segundo previniendo la desestructuración y disfuncionabilidad familiar, que hoy en día, se constituyen como problemas sanitarios, pero que están revestido de invisibilidad para los efectores de las políticas públicas.

Cuidar del barrio, de la institución educativa, de los centros laborales, de la comunidad, etc., es un reto importante para nosotros los salubristas. Castellanos (1992) define intervenciones en salud pública, nosotros la denominaremos dimensiones en un intento de hacer tangible el cuidado del colectivo, su planteamiento se sustenta en la necesidad de cuidar interviniendo el modo de vida, la condición de vida, y los estilos de vida para garantizar desarrollo social.

Cuidar del modo de vida, requiere conocer su cultura, sus legítimas aspiraciones, el posicionamiento de la persona-colectivo en la estructura social, su representación social, como interactúa con el medio ambiente. Esta información es básica para cuidar con el colectivo y para el colectivo. Otra dimensión a intervenir, no menos importante lo cons- 
tituye la condición de vida, conocer las formas de relacionamiento al interno y externo de su colectivo, los roles, el poder, etc., son de grande ayuda para comprender y diseñar el proceso de intervención cuyo objetivo es establecer nuevas estructuras de poder con mayores ventajas.

Promover estilos de vida saludables va más allá de la adopción de formas de comportamiento saludables planteadas por Lalonde (Ministerio de Salud de México, 1986), significa dotar de estructura para hacer objetivo el comportamiento saludable. Muchas veces educamos a las madres o cuidadoras del hogar sobre cómo prevenir las enfermedades diarreicas a través de la higiene de manos y de alimentos; olvidamos que su condición de vida no permite tener agua segura, elemento necesario para la higiene, mucho menos de una congeladora para conservar su alimento.

La comunidad aun es un espacio para crear y recrear formas de cuidado con el sujetoobjeto colectivo. Abrigamos la esperanza que los consultorios de enfermería se constituirán no solo en ese espacio físico sino también virtual donde se proporcione y se fomente el auto-cuidar y el ejercicio pleno de autonomía. Sin duda alguna estos se constituyen en elementos básicos para generar desarrollo.

Existe una agenda pendiente, que está relacionada con todas las iniciativas de lucha por un ejercicio autónomo de la profesión. En el pensamiento gramsiano, se denomina lucha contrahegemónica cuando una categoría profesional, en este caso Enfermería, implementa un proceso de lucha por reivindicaciones gremiales y sociales; y busca convertirse en Estado (Gramsci, 2007).

Vemos un grande desafío para la universidad, en la medida que es la responsable de crear y recrear conocimiento experto que sustente un ejercicio profesional inteligente y autónomo; requiere entonces acelerar, profundizar cambios estructurales y curriculares que aseguren calidad en la formación de nuevos profesionales. Además, necesitamos consolidar el aporte de las escuelas de posgrado y postítulo para la autonomía profesional.

Con optimismo y sin desmayo requiere continuar procesos de advocacy con los formuladores y operadores de las políticas públicas a fin de explicitar en términos legales y estructurales la legítima aspiración de la categoría profesional de enfermería de ofertar cuidados profesionales con visas al desarrollo humano y local. Ayudaría mucho tener representación en el poder legislativo y consolidar la presencia sanitaria del enfermero en el ejecutivo

Los enfermeros(as) requerimos entusiasmo y mucha energía para el trabajo que se avizora, hay una grande expectativa social y no defraudaremos, necesitamos trabajar en el sistema sanitario, creando y recreando conocimiento, métodos, técnicas que hagan tangible el producto a ofertar (cuidado de enfermería) en los consultorios público-privados.

Precisamos establecer nuevas formas de relacionamiento con las diferentes categorías profesionales y técnicos de salud, diferenciando y posicionando nuestro producto en el mercado sanitario, sin duda requiere el concurso y compromiso de los más de 80000 enfermeros distribuidos en el ámbito nacional. El Colegio de Enfermeros como ente encargado de velar por el ejercicio profesional autónomo debe crear y fortalecer comisiones 
de trabajo a nivel político, técnico-científico, académico y asistencial a fin de canalizar las diferentes iniciativas.

El horizonte es prometedor, mas hoy, parafraseando al poeta y escritor peruano César Vallejo, considerado uno de los mayores exponente de las letras en el Perú y el mundo, "¡hermanos ENFERMEROS(AS), aún hay mucho por hacer!"

\section{REFERENCIAS BIBLIOGRÁFICAS}

Amartya Sem. (2008). Libertad y desarrollo. Barcelona: Editorial Planeta AS.

Polo Campos, F. H. (2009). Resposta social das políticas públicas de saúde na perspectiva dos idosos: contribuições da enfermagem-estudo comparado Brasil / Perú. Río de Janeiro: UFRJ/ EEAN. Tese (Doutorado em Enfermagem) - UFRJ/EEAN/ Programa de Pósgraduação. Orientadora Regina Célia Gollner Zeitouneem.

Polo Campos, F. H. (mayo 2010). Fundamentos para el modelo de cuidado integral, de calidad y universal. Consultado el 24-09-2015. Disponible en: https://www.google. com.br/webhp?sourceid=chrome-instant\&ion=1\&espv=2\&ie=UTF-8\#q=fredy+her$\underline{\text { nan }+ \text { polo+campos\&start }=20 \text {. }}$.

Hatton, E. J. (1988). Teacher`s work as bricolaje: Implications for teacher education. British Journal of sociology of education. Mencionado en: Iglesias Salvador José. (Enero-abril, 1995). Autonomía profesional: entre el reto y la realidad. Revista Interuniversitaria de Formación del Profesorado, 22, 89-98. Consultado el 23-09-2015. Disponible en: http://www.aufop.com/aufop/uploaded files/articulos/1269129496. pdf.

Pérez A. J. (1993). Autonomía profesional y control democrático. Cuadernos de pedagogía, 220, 25-30. Mencionado en: Iglesias Salvador José. (Enero-abril, 1995). Autonomía profesional: entre el reto y la realidad. Revista Interuniversitaria de Formación del Profesorado, 22, 89-98. Consultado el 23-09-2015. Disponible en: http://www.aufop. com/aufop/uploaded files/articulos/1269129496.pdf

Iglesias Salvador José. (Enero-abril, 1995). Autonomía profesional: entre el reto y la realidad. Revista Interuniversitaria de Formación del Profesorado, 22, 89-98. Consultado el 23-09-2015. Disponible en: http://www.aufop.com/aufop/uploaded files/articulos/1269129496.pdf

Cárnio E. C. (Julio-agosto 2013). Consultorios de enfermería [Editorial]. Rev. Latino-Am. Enfermagem [Internet]. Consultado el 23-09-2015]; 21(4). Disponible en: http:// www.scielo.br/pdf/rlae/v21n4/es 0104-1169-rlae-21-04-0833.pdf.

OMS. (2008). La atención primaria de la salud. Más necesaria que nunca. Informe sobre la salud en el mundo. Ginebra: OMS. Consultado el 25/3/2010. Disponible en: www. who.int/whr/2008/08_report_es.pdf. 154 pp. 
Heideguer. (1969). Ser e tempo. Mencionado en: Waldow, V. R. (2006). Cuidar: expressão humanizadora da enfermagem. Petrópolis: Vozes.

Frank C., Hobbs N., Stewart J. (1998). Rehabilitation on Palliative care units: Case discussion, Journal of Palliative care, 14 (2): 50-53.

Astudillo W. y Mendinueta C. (s. f.). La rehabilitación de los cuidados paliativos. Centros de Salud de Bidebieta- la Paz, San Sebastián y Astigarraga, Gipuzkoa.

Ministerio de Salud de México. (1986). Carta de Otawa. [Internet]. Consultado el 2309-2015. Disponible en: http://www.promocion.salud.gob.mx/dgps/descargasl/ promocion/2 carta de ottawa.pdf

Castellanos R., (1998). Redes y sociedad. Mencionado en: Guareschi, P. A. Sociologia da prática social. Petrópolis, Río de Janeiro: Vozes, 1992.

Gramsci, A. (2007). Cadernos do cárcere. Río de Janeiro: Civilização Brasileira. Volume 3 (1891-1937). 